\title{
Adaptive OFDM-IM System Over Faded Shadowing Channel
}

\author{
Ibrahim Mokhtar Aboharba, Quazi Mehbubar Rahman, Raveendra Rao
}

Department of Electrical and Computer Engineering, Western University, London, Canada

Email address:

iaboharb@uwo.ca (I. M. Aboharba),qrahman3@uwo.ca (Q. M. Rahman),rrao@uwo.ca (R. Rao)

\section{To cite this article:}

Ibrahim Mokhtar Aboharba, Quazi Mehbubar Rahman, Raveendra Rao. Adaptive OFDM-IM System over Faded Shadowing Channel. American Journal of Networks and Communications. Vol. 8, No. 1, 2019, pp. 32-46. doi: 10.11648/j.ajnc.20190801.14

Received: April 25, 2019; Accepted: May 30, 2019; Published: July 17, 2019

\begin{abstract}
Spectral efficiency (SE) and energy efficiency (EE) play major roles in evaluating the quality of service (QoS) of a wireless communication system. Designing an efficient wireless communication system requires trade-off between these two parameters. Orthogonal frequency division multiplexing technique with Index Modulation (OFDM-IM) has been introduced in the literature to increase the SE compared to traditional OFDM. In this paper, an adaptive technique with OFDM-IM is introduced in an M-QAM based scheme to maintain a guaranteed level of average bit error probability (ABEP) with enhanced SE. It has been demonstrated that by adaptively varying the number of active subcarriers and modulation levels in M-QAM scheme, maximum EE can also be achieved for an acceptable ABEP. The closed-form expressions of ABEP and pairwise error probability (PEP) for OFDM-IM with M-ary QAM modulation are derived and examined over the composite Nakagami-m Gamma (NG) fading channel model using greedy detection (GD). In addition, the performance of adaptive OFDM-IM with M-QAM Modulation scheme is evaluated in terms of efficiency metrics, outage probability, and ABEP. The obtained results show that the adaptive scheme offers high potential for accomplishing significant improvement in SE and EE while maintaining acceptable ABEP even under severe channel impairment.
\end{abstract}

Keywords: Orthogonal Frequency Division Multiplexing (OFDM), Index Modulation (IM), Pairwise Error Probability (PEP), Adaptive Modulation

\section{Introduction}

In recent years, an exponential growth in demand for high data-rate transmission has shifted the focus in the orthogonal frequency division multiplexing (OFDM) scheme and its variants in the wireless communication system. The OFDM scheme has been in the forefront of the majority of wireless communication systems due to its high data-rate, transmission capability, immunity, and robustness to multipath fading, and more [1]. One variant of OFDM scheme, known as OFDM with index modulation (OFDM-IM) was proposed by combining the concept of index modulation (IM) with conventional OFDM system, [2-3]. In OFDM-IM system, ON-OFF keying modulation is employed to convey additional information by activating and deactivating the subcarriers. The OFDM-IM scheme reduces the bit error rate (BER) over traditional OFDM schemes, and it offers a better trade-off between spectral and energy efficiency [1]. A generalized OFDM-IM scheme is introduced as OFDM-GIM, [4]. The active subcarriers are no longer fixed in OFDM-GIM, which further enhances spectral efficiency with an increase in BER. The tight upper bound BER of OFDM-IM is proposed over Additive White Gaussian Noise (AWGN) channel, which includes the effects of both index and M-ary modulation, [5]. A low complexity detection method, called greedy detection (GD), is introduced to reduce the complexity of the receiver over Maximum Likelihood (ML) detection, [6]. The GD is utilized in two processes: the location of the active subcarriers and the modulated data symbols. Each process is preformed independently. The first process detects the active subcarriers by measuring the received signal power on every subcarrier. The subcarriers, which have higher received power than a preset threshold value, are estimated as ones. Whereas, the subcarriers, which have lower received power, are estimated as zeros. The second process, the Maximum-Likelihood (ML) is applied to estimate the data symbols in the active subcarriers [6]. Pairwise error probability (PEP) with the GD 
method is addressed over two-wave with diffuse power (TWDP) fading channels for device-to-device (D2D) wireless systems, [7]. The TWDP fading channel has been used to analyze different scenarios, including Rayleigh fading. More recently, a multi-path fading scenario has been considered with the GD method in OFDM-IM using Nakagami-m fading channel, [8]. Also, the BER of M-ary QAM and PSK modulations in OFDM-IM scheme has been derived with different power allocation policies over Nakagami-m fading channel, [9]. As a result, it is worthwhile to examine the performance of OFDM-IM over a variety of fading scenarios. In all the aforementioned research works, the focus was on the multipath fading and line of sight (LOS) scenarios, where the effect of shadowing was not explicitly addressed. To get an overall view of the wireless channel impairments and their effects in OFDM-IM system, a composite Nakagami-m and Gamma (NG) channel model have been studied in this paper. The NG channel model is chosen because it can be modeled in to a large number of shadowing and fading channel conditions from moderate to severe scenarios in the system. In this study, several techniques have been introduced to improve the performance of an OFDM-IM based wireless communication systems over these fading channel conditions. One of these techniques is adaptive modulation, which is employed to enhance spectral efficiency (SE) over wireless channels based on fading and shadowing [10]. We are proposing adaptive modulation for an OFDM-IM based system to maximize the energy efficiency (EE) along with the SE by adapting different M-QAM constellation sizes and active subcarriers of OFDM-IM system. Here the impact of wireless channels are studied using the greedy detection method, and numerical closed-form expressions have been derived for average pairwise error probability (PEP), and average bit error probability (ABEP). Also, $\mathrm{ABEP}, \mathrm{SE}$, and $\mathrm{EE}$ of adaptive OFDM-IM with M-QAM modulation are investigated and evaluated over composite fading and shadowing conditions. The rest of this paper is arranged as follows: In Section 2, the system model of OFDM-IM, the performance analysis of OFDM-IM including pairwise error probability (PEP) and average bit error probability (ABEP) are presented over the composite fading and shadowing channel model. The adaptive M-QAM system with OFDM-IM and its associated performance over NG channel along with numerical results and discussion are presented in Section 3. Finally, Section 4 concludes this paper.

\section{Performance Analysis of OFDM-IM System}

\subsection{System Model of OFDM-IM}

The block diagram of the OFDM-IM transceiver is shown in Figure 1, where the dashed feedback path has been included to represent the adaptive modulation scheme that is discussed in Section 3 [3]. At the transmitter side, the total number of data bits $D_{t}$ are entered into the OFDM-IM transmitter to form one OFDM block symbol. Every block of $D_{t}$ bits is divided into $g$ groups, each consisting of $p$ bits, i.e., $D_{t}=p g$. Each group of $p$ bits is assigned to one of the $g$ OFDM sub-blocks. The length of the sub-bock is $n$, where $n$ $=N / g$ and $N$ represents the total number of subcarriers. In a conventional OFDM system, all $N$ subcarriers are active and each subcarrier conveys M-ary QAM signal constellation symbols, whereas in OFDM-IM, only $k$ out of $n$ subcarriers per group are active. The subcarriers, which are active, transmit Mary QAM signal constellation symbols, and the inactive $(n-k)$ subcarriers, are zero padded. Thus, no data are conveyed by inactive subcarriers. The number of active subcarriers $(k)$ should be smaller or equal to the length of a sub-block $(n)$, i.e., $k \leq n$. Therefore, $p$ bits are split into two parts; the first part contains $p_{1}$ bits and the second part contains $p_{2}$ bits, i.e., $p=p_{1}+p_{2}$ as illustrated in Figure 1 . The maximum number of possible active subcarrrier combinations is found by the binomial coefficient of $C(n, k)=$ $n ! / k !(n-k) !$, which modulates $p_{1}=\left\lfloor\log _{2} C(n, k)\right\rfloor$, where $\lfloor$. is floor function, i.e., the largest lower integer number. $p_{I}$ bits are employed to map the indices of the subcarriers that transmit QAM symbols, and $p_{2}=k \log _{2} M$ bits are mapped to sub-block, i.e., $s_{\beta}=\left[s_{\beta}(1), \cdots, s_{\beta}(N)\right]$, where $\beta=1, \cdots, g$ and $i$ $=1, \cdots, k . \quad D_{1}=p_{1} g=\left\lfloor\log _{2} C(n, k)\right\rfloor g$ is total number of bits that are conveyed by the $k$ active sub-carriers in each OFDM block. $D_{2}=p_{2} g=\left(k \log _{2} M\right) g$ indicates the total number of bits that are transmitted by $k \mathrm{M}$-ary QAM symbols. The total number of bits that can be transmitted by one OFDM-IM block symbol is $D_{t}=D_{1}+D_{2}$ [3]. After OFDM-IM block is formed, IFFT is applied. Then, cyclic prefix is added and the modulated data is sent over the proposed NG fading and shadowing channel model. The NG model is described in next section. At receiver side, the received signal of the OFDM-IM scheme is characterized as $y=s H+w$, where $y$ $=[y(1), \ldots, y(N)], s$ is an OFDM-IM transmitted signal vector, $H=\operatorname{diag}[h(1), \ldots, h(N)]$ denotes the channel fading coefficient matrix, and $w=[w(1), \ldots, w(N)]$ is additive white Gaussian noise (AWGN) vector. After the cyclic prefix is removed, the FFT is applied to the received signal. Then, the GD is applied to detect the index of the subcarriers. An inactive subcarrier is estimated to be 0 and an active subcarrier are estimated to be 1. The de-modulator is employed in every active subcarrier to estimate the received signal. Finally, estimated signal is reformed from parallel to serial stream.

\subsection{Composite Model of Fading and Shadowing Channel}

Wireless channels suffer from short-term and long-term fading. The short-term fading, also known as multipath fading, is modeled by the Nakagami-m distribution and the long-term fading, also known as shadowing, is described using Gamma 


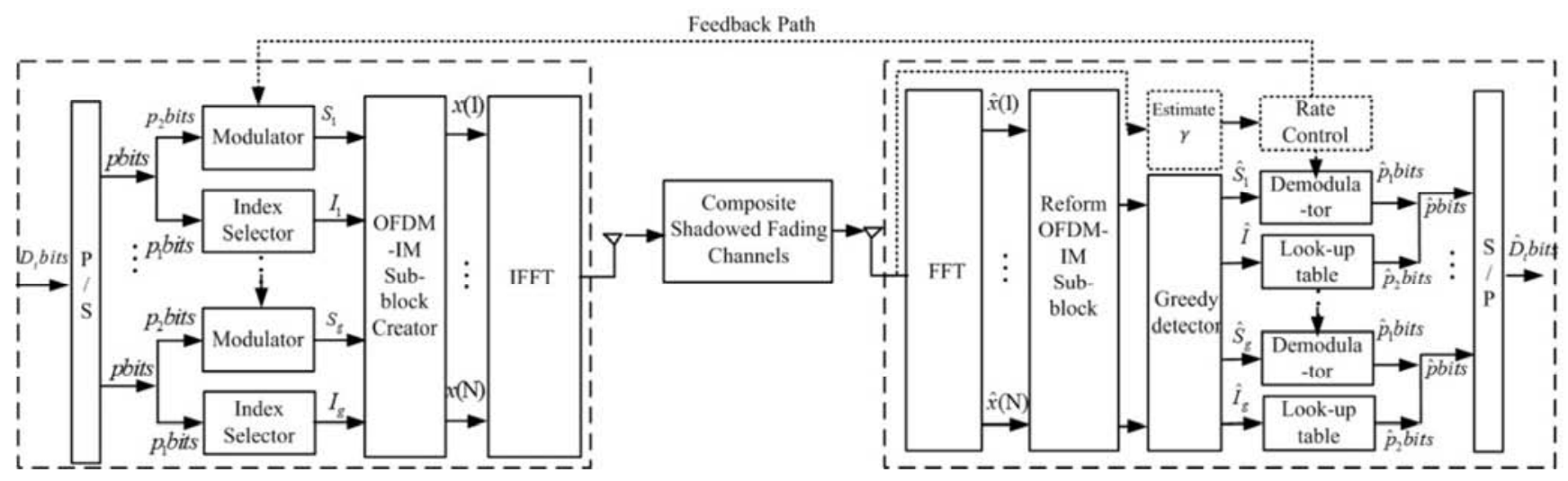

Figure 1. OFDM-IM Transceiver (The dashed feedback path is a part of the adaptive modulation scheme).

distribution. Generally, the probability density function (PDF) of the channel gain $\alpha$ is expressed as [11]:

$$
f_{\alpha}(\alpha \mid \Omega)=\frac{2 m^{m} \alpha^{2 m-1}}{\Omega^{m} \Gamma(m)} \exp \left(-\frac{m \alpha^{2}}{\Omega}\right), \quad m \geq \frac{1}{2}
$$

where $\Gamma(\mathrm{m})$ is the gamma function with $\Gamma(m)=(m-1)$ !, $\Omega=\mathrm{E}\left(\alpha^{2}\right)$ is the average received power gain, and $m=\Omega / \mathrm{E}\left[\left(\alpha^{2}-\Omega^{2}\right)\right]$ defines the fading factor of the channel. In (1), $m=1$ matches the Rayleigh distribution, $m>1$ matches the Rician distribution, and $m<1$ corresponds to severe fading. The conditional PDF of Nakagami-m channel model with respect to Signal to Noise Ratio (SNR), $\gamma$, is given by [11]:

$$
P_{\gamma}\left(\gamma \mid \gamma_{f}\right)=\frac{m^{m} \gamma^{m-1}}{\gamma_{f}^{m} \Gamma(m)} \exp \left(-\frac{m \gamma}{\gamma_{f}}\right)
$$

where $\gamma$ is the instantaneous received SNR and $\gamma_{f}$ is the average received SNR, when there is no shadowing effect. The presence of shadowing causes the average received $\operatorname{SNR}\left(\gamma_{f}\right)$ in PDF of Nakagami-m to be random. The Nakagami-m distribution is combined with Gamma distribution for more generalized estimation a channel that is effected by both shadowing and fading. When the shadowing is present, the envelope of the signal follows the Nakagami-m distribution, and the power of the envelope follows the Gamma distribution. The PDF of Gamma distribution is expressed as [12-13]:

$$
P_{\gamma_{f}}\left(\gamma_{f}\right)=\frac{1}{\Gamma(c)}\left(\frac{c}{\bar{\gamma}}\right)^{c} \gamma_{f}^{c-1} \exp \left(-\gamma_{f} \frac{c}{\bar{\gamma}}\right)
$$

where $\gamma_{f}$ is the instantaneous received SNR, $\bar{\gamma}$ is the average received SNR and $c$ is the shadowing factor. Therefore, the combined PDF of shadowing and fading is accomplished by averaging the conditional PDF of Nakagami-m over the PDF of Gamma, and it is expressed as [13]:

$$
P(\gamma)=\int_{0}^{\infty} P\left(\gamma \mid \gamma_{f}\right) P_{\gamma_{f}}\left(\gamma_{f}\right) d \gamma_{f}
$$

The PDF of combined Nakagami-m and Gamma (NG) model is also known as generalized $\mathrm{k}\left(\mathrm{k}_{\mathrm{G}}\right)$ model and the PDF of NG model can be obtained by substituting (2) and (3) into (4) as [13]:

$$
P(\gamma)=\frac{c^{c} m^{m} \gamma^{m-1}}{\Gamma(c) \Gamma(m) \bar{\gamma}^{c}} \int_{0}^{\infty} \gamma_{f}^{c-m-1} e^{\frac{m \gamma}{\gamma_{f}}-\frac{c \gamma_{f}}{\bar{\gamma}}} d \gamma_{f}
$$

By using the Table of Integrals, the closed-form expression of NG model is formulated as [14, (3.471.9), 16]:

$$
P(\gamma)=\frac{2}{\Gamma(c) \Gamma(m)}\left(\frac{c m}{\bar{\gamma}}\right)^{\frac{c+m}{2}} \frac{c+m-2}{\gamma^{2}} K_{c-m}\left(2 \sqrt{\frac{c m \gamma}{\bar{\gamma}}}\right)
$$

where the $\mathrm{c}$ and $\mathrm{m}$ are the shadowing and fading parameters of the composite NG model, respectively. $K_{c-m}($.$) is the$ modified Bessel function of order $(c-m)$. Here, it can be shown that $\int_{0}^{\infty} P(\gamma) d \gamma=1$. The NG model can characterize various shadowing and fading channel models by employing different combinations of $\mathrm{c}$ and $\mathrm{m}$. For instance, when $\mathrm{c}$ approaches infinity, the NG model approximates the Nakagami-m model. $\mathrm{m}=1$ describes the Rayleigh log-normal (R-L) model. When $\mathrm{c}$ and $m$ increase, the effects of shadowing and fading decreases. As $\mathrm{c}$ and $\mathrm{m}$ approach infinity, the NG model approaches Additive White Gaussian Noise (AWGN) channel model.

\subsection{Analysis of Pairwise Error Probability (PEP)}

In this section, using the greedy detection method the PEP of OFDM-IM for M-ary QAM schemes is evaluated under shadowing and fading, which is modeled by Nakagami-m-Gamma in wireless. As mentioned earlier, the greedy detector involves two processes. The first process detects the indices of the active subcarriers (identified by index 1) and inactive subcarriers (identified by index 0 ). The second process modulates a data symbol at the active subcarriers by employing the Maximum-Likelihood (ML) detection method. When the active subcarrier index $(\delta)$ is 
incorrectly estimated at receiver as inactive index $(\hat{\delta})$, this event is known as the pairwise error event (PEE), i.e., $(\delta \rightarrow \hat{\delta})$ and $\delta \neq \hat{\delta}$. The conditional pairwise error probability (PEP) can be described as the probability having the PEE, $\operatorname{PEP}(\delta \rightarrow \hat{\delta})$, when the channel information is available at receiver. The PEP has been derived as [6]:

$$
P E P(\delta \rightarrow \hat{\delta})=1-\sum_{q=0}^{n-k}\left(\begin{array}{c}
n-k \\
q
\end{array}\right) \frac{-1^{q}}{q+1} \exp \left(\frac{-q \gamma_{\delta}}{q+1}\right)
$$

where $\gamma$ is the instantaneous signal to noise ratio (SNR) of the active subcarrier. The approximate expression of $P E P$ has been derived as [8]:

$$
P E P(\delta \rightarrow \hat{\delta}) \simeq \frac{n-k}{2} e^{-\frac{\gamma_{\delta}}{2}}
$$

The total probability of the upper bound for PEP for OFDM-IM is given by [7]:

$$
\begin{aligned}
& P E P_{t} \leq \frac{k}{n} \sum_{\delta=1}^{n} \operatorname{PEP}(\delta \rightarrow \hat{\delta}) \\
& \leq k \cdot P E P(\delta \rightarrow \hat{\delta})
\end{aligned}
$$

For simplicity on notations, the pairwise error probability of subcarriers $P E P(\delta \rightarrow \widehat{\delta})$ is identified by $P E P$ and the SNR of the active subcarriers $\gamma_{\delta}$ is identified by $\gamma$ from hereafter. The average PEP for OFDM-IM over the shadowed fading channel can be calculated by averaging (7) over (6):

$$
\begin{aligned}
& P E P_{N G}=\int_{0}^{\infty}\left[1-\sum_{q=0}^{n-k}\left(\begin{array}{c}
n-k \\
q
\end{array}\right) \frac{-1^{q}}{q+1} \exp \left(\frac{-q \gamma}{q+1}\right)\right] \\
& \times \frac{2}{\Gamma(c) \Gamma(m)}\left(\frac{c m}{\bar{\gamma}}\right)^{\frac{c+m}{2}} \gamma^{\frac{c+m-2}{2}} K_{c-m}\left(2 \sqrt{\frac{c m}{\bar{\gamma}}} \gamma\right) d \gamma
\end{aligned}
$$

Using the Table of Integrals in (10), the average analytical expression of PEP can be expressed as [14, (6.643.3)]:

$$
\begin{aligned}
P E P_{N G}=1-(\beta)^{v} & \sum_{q=0}^{n-k}\left(\begin{array}{c}
n-k \\
q
\end{array}\right) \frac{-1^{q}}{q+1} e^{\frac{\beta(q+1)}{2 q}}\left(\frac{q}{q+1}\right)^{-v} \\
& \times W_{-v, l}\left(\frac{\beta(q+1)}{q}\right)
\end{aligned}
$$

where, $\beta=\frac{c m}{\bar{\gamma}}, \nu=\frac{c+m-1}{2}, \imath=\frac{c-m}{2} \mathrm{~m}$ and $W($.$) is the Whittaker$ function.

By Substituting (11) into (9), the overall PEP over the shadowed and fading channel is formulated as:

$$
\begin{aligned}
& P E P_{t, N G}=k\left[1-(\beta)^{v} \sum_{q=0}^{n-k}\left(\begin{array}{c}
n-k \\
q
\end{array}\right) \frac{-1^{q}}{q+1} e^{\frac{\beta(q+1)}{2 q}}\left(\frac{q}{q+1}\right)^{-v}\right. \\
& \left.\times W_{-v, l}\left(\frac{\beta(q+1)}{q}\right)\right]
\end{aligned}
$$

By following this approach, the approximate PEP for OFD M-IM over the shadowed and fading channel is found by averaging (8) over (6) as:

$$
\begin{aligned}
& P E P_{N G} \simeq \int_{0}^{\infty} \frac{n-k}{2} e^{\frac{\gamma}{2}} \times \frac{2}{\Gamma(c) \Gamma(m)}\left(\frac{c m}{\bar{\gamma}}\right)^{\frac{c+m}{2}} \gamma^{\frac{c+m-2}{2}} \\
& K_{c-m}\left(2 \sqrt{\frac{c m}{\bar{\gamma}}} \gamma\right) d \gamma
\end{aligned}
$$

The approximate closed-form expression for PEP can be obtained by applying the Table of Integrals in (13) as [14, (6.643.3)]:

$$
P E P_{N G}^{a p p o x} \simeq \frac{n-k}{2}(\beta)^{v} e^{\beta}\left(\frac{1}{2}\right)^{-v} W_{-v, l}(2 \beta)
$$

The approximation of overall $P E P$ is determined by substituting (14) into (9) as:

$$
P E P_{t, N G}^{a p p o x} \simeq k\left[\frac{n-k}{2}(\beta)^{v} e^{\beta}\left(\frac{1}{2}\right)^{-v} W_{-v, l}(2 \beta)\right]
$$

\subsection{Average Bit Error Probability (ABEP)}

The bit error rate $\left(P_{e M}\right)$ of OFDM-IM is a result of mis-detection in the active subcarrier and M-ary symbol. This mis-detection appears in three errored situations. The first one results from the mis-detection in the index of the active subcarrier and M-ary symbol, and it is given as $e_{I M I}=P E P . P_{e M}$. The second one results from the mis-detection of the index of the active sub-carrier, but correct detection of the M-ary symbol, and it is expressed as $e_{I M 2}=P E P$. $\left(1-P_{e M}\right)$. The third one results from the correct detection of the index of the active sub-carrier, but the mis-detection of the M-ary symbol, and it is defined as $e_{I M 3}=(1-P E P) P_{e M}$. Therefore, the total bit error probability $(B E P)$ can be obtained as $B E P=P E P+(1-P E P) P_{e M}$, where $B E P$ is the bit error probability over the AWGN channel of the M-ary modulation scheme [5]. The total overall BEP expression can be written as:

$$
\begin{aligned}
& B E P_{t} \leq \frac{k}{n} \sum_{\delta=1}^{n}\left[P E P+(1-P E P) P_{e M}\right] \\
& \leq k\left[P E P+(1-P E P) P_{e M}\right] \\
& \leq k\left[P E P-P_{e M}+P E P \cdot P_{e M}\right]
\end{aligned}
$$

The BER of coherent M-ary QAM over the AWGN channel with gray coding is given by [10]: 


$$
P_{e M} \simeq 0.2 \exp \left(\frac{-3 \gamma}{2(M-1)}\right)
$$

where $\gamma$ indicates instantaneous SNR and $M$ denotes modulation level.

Averaging (16) over (6) and inserting (7), (8) and (17) into (16), the upper bound and approximate average $B E P(A B E P)$ of OFDM-IM over the shadowing and fading channel can be found as:

$$
A B E P_{K_{G}}=\int_{0}^{\infty} B E P \cdot P_{\gamma}(\gamma) d \gamma
$$

The upper bound and approximate closed form expressions of the M-ary QAM-OFDM-IM scheme for the greedy detection can be evaluated as shown in (19) and (20), respectively:

$$
\begin{gathered}
A B E P_{K_{G}}^{Q A M} \leq K\left[1-(\beta)^{v} \sum_{q=0}^{n-k}\left(\begin{array}{c}
n-n \\
q
\end{array}\right)\right. \\
\frac{-1^{q}}{q+1} e^{\frac{\beta}{2 L}}(L)^{-v} W_{-v, l}\left(\frac{\beta}{L}\right) \\
+0.2(\beta)^{v} \sum_{q=0}^{n-k}\left(\begin{array}{c}
n-n \\
q
\end{array}\right) \frac{-1^{q}}{q+1} e^{\frac{\beta}{2\left(L+\frac{1}{\alpha}\right)}} \\
\left.\left(L+\frac{1}{\alpha}\right)^{-v} W_{-v, l}\left(\frac{\beta}{L+\frac{1}{\alpha}}\right)\right] \\
A B E P_{\mathrm{t}, \mathrm{NG}}^{a p p o x, Q A M} \leq k\left[\frac{n-k}{2}(\beta)^{v} e^{\beta}\left(\frac{1}{2}\right)^{-v}\right. \\
(2 \beta)+0.2(\beta)^{v} e^{\frac{\beta \alpha}{2}}\left(\frac{1}{\alpha}\right)^{v} W_{-v, l}(\beta \alpha) \\
\left.-\frac{n-k}{10}(\beta)^{v} e^{\frac{\beta}{2 D}}(D)^{-v} W_{-v, l}\left(\frac{\beta}{D}\right)\right]
\end{gathered}
$$

where the parameters are $\beta=\frac{c m}{\bar{\gamma}}, L=\frac{q}{q+1}, \quad \alpha=\frac{2(M-1)}{3}$, $v=\frac{c+m-1}{2}, \quad l=\frac{c-m}{2}$ and $D=\frac{M+2}{M-1}$. The terms $c, m$ and $M$ are the shadowing factor, the fading factor and the constellation size, respectively.

\subsection{Simulation of ABEP}

In this section, the ABEP of OFDM-IM is obtained using Monte Carlo simulation, which is accomplished using Matlab for greedy detection over the shadowing and fading channel. The channel gain $\left(h_{k G}\right)$, which describes shadowing and fading, can be generated as [16-17]:

$$
h_{K_{G}}=2 \sqrt{\frac{\left(G_{m} \times G_{c}\right)}{|c-m|+1}}
$$

where $G_{m}$ and $G_{c}$ denote the pdf of Gamma distribution for the fading $(m)$ and shadowing $(c)$ factors, respectively. For the AWGN channel, the channel parameters $m$ and $c$ are set to infinity to derive channel gain $\left(h_{K G}=1\right)$. In contrast, for the Rayleigh channel, the channel parameters are set to $(m=1)$ and $(c=\infty)$. The simulation scheme is formed from two parts, the transmitter and the receiver as follows: at the transmitter end, (see Figure 1 for clarity), serial random data stream $\left(D_{t}\right)$ is generated and divided into $g=N / n$ groups. In each group, $p$ bits are split into two sub-groups $p_{1}$ and $p_{2}$, relying on $n$ and $k$ as demonstrated in Section 2. A look-up table $(L U T)$ is used to map each sub-block. For instance, the $L U T$ for $n=4, k=2$ and $p_{1}$ $=2$ is shown in Table 1 .

Table 1. LUT for $n=4$ and $k=2$.

\begin{tabular}{ll}
\hline $\mathbf{P}_{\mathbf{1}}$ bits & Sub-block \\
\hline 00 & 1010 \\
01 & 0101 \\
10 & 1001 \\
11 & 0110 \\
\hline
\end{tabular}

Here, $p_{1}$ bits are employed to indicate the active subcarrier and the $p_{2}$ bits are mapped to the active subcarrier to create one of the M-ary Modulation symbols in the sub-block. The OFDM-IM sub-block creator (see Figure 1 for clarity) combines g sub-blocks to create one OFDM-IM symbol. This symbol is passed through invers fast Fourier transform (IFFT), and then a cyclic prefix is applied. The fading and shadowing channel $\left(h_{K G}\right)$ parameters are in Table 2.

Table 2. Simulation Parameters.

\begin{tabular}{ll}
\hline Parameter & Value \\
\hline FFT Size & 64 \\
Guard interval & 16 \\
Length of Sub-block (n) & 4 \\
Number of Active Subcarrier & 2 \\
Fading and Shadowing Factors & $1,2,3$, and 4 \\
Number of Symbols & 10000 \\
Castellation Size & 4 and 16 QAM \\
SNR & 0 dB to 40dB \\
\hline
\end{tabular}

On the receiver side (see Figure 1 for clarity), two processes are required to predict the index modulation and M-ary modulation symbol using greedy detection. In the first step, the received subcarriers in every sub-block that have the largest power are estimated to be 1, i.e., active. The remaining bits, which have low received power, are estimated to be 0 , i.e., inactive. $\hat{p}_{1}$ bits are retrieved by the known LUT at the receiver that is employed at the transmitter. In the second step, ML decision is applied to estimate $\hat{s}$ in every active subcarrier, i.e., $\hat{p}_{2}$. Finally, $\hat{p}_{1}$ and $\hat{p}_{2}$ are integrated to compose $\hat{p}$ and the original data stream is reshaped from parallel to serial stream as $\hat{D}$. The average PEP for BPSK is plotted in Figure 2 over the composite Nakagami-m and Gamma (NG) model for various fading $(m)$ and shadowing (c) parameters. The average PEP for BPSK is plotted in Figure 2 over the composite Nakagami-m and Gamma (NG) model for various fading $(m)$ and shadowing $(c)$ parameters. The results validates the analytical result that is presented in $[8$, 10]. From Figure 2, it is observed that the composite fading and shadowing channel approximately matches the Rayleigh and Nakagami-m $(m=1)$ fading channels at $c=2.15$ and $m=1$. 
Figure 3 and Figure 4 illustrate $B E P$ for 4 and 16 QAM factors, respectively. modulation schemes with various fading and shadowing

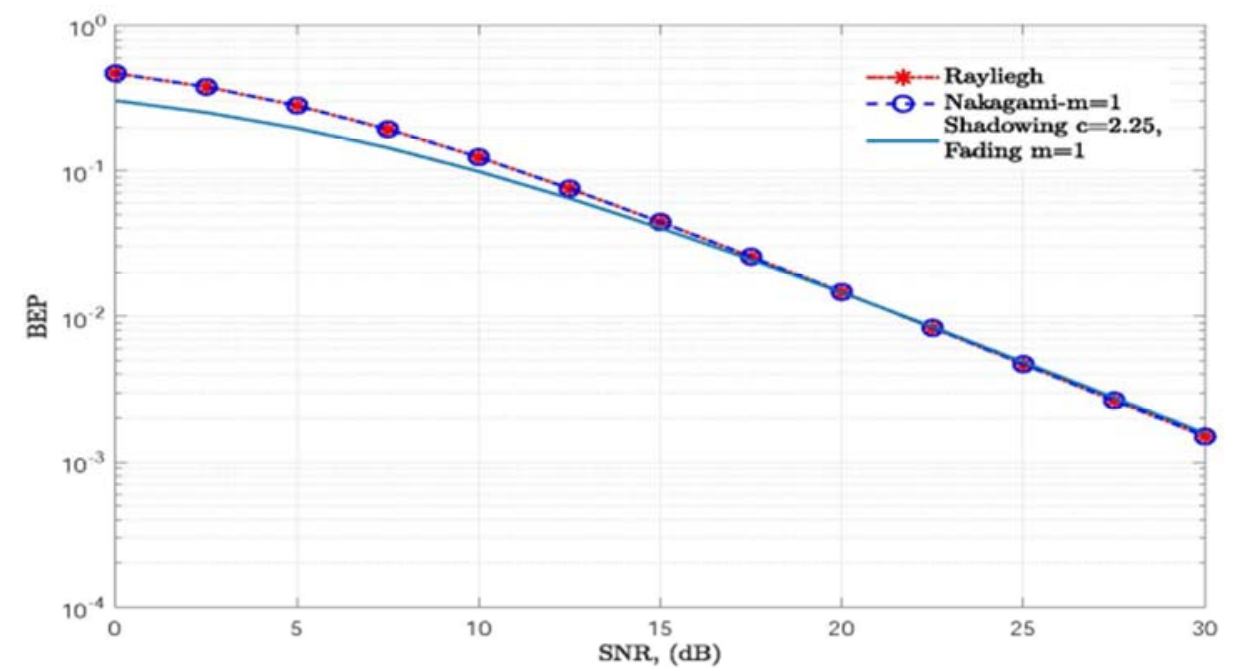

Figure 2. Comparison of average PEP of OFDM-IM with greedy detection over the different fading channels as the faction of SNR.

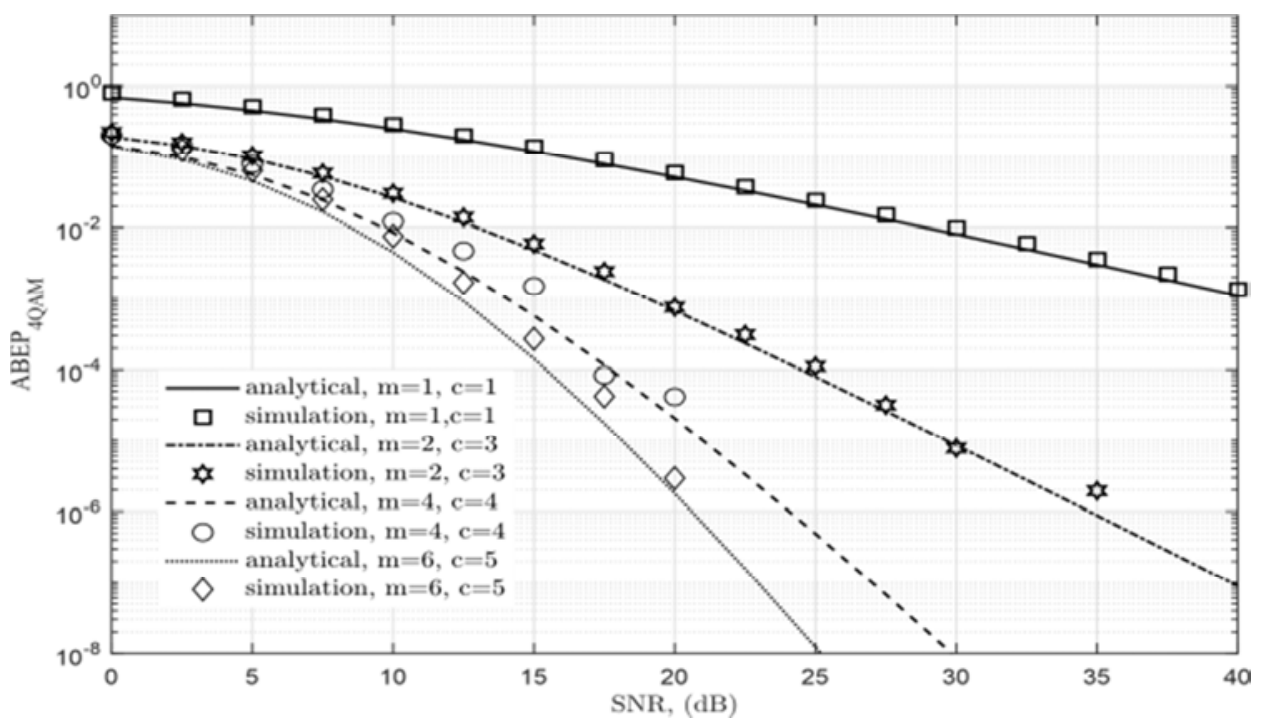

Figure 3. BEP for OFDM-IM with 4-QAM as a function of SNR over various shadowing and fading parameters.

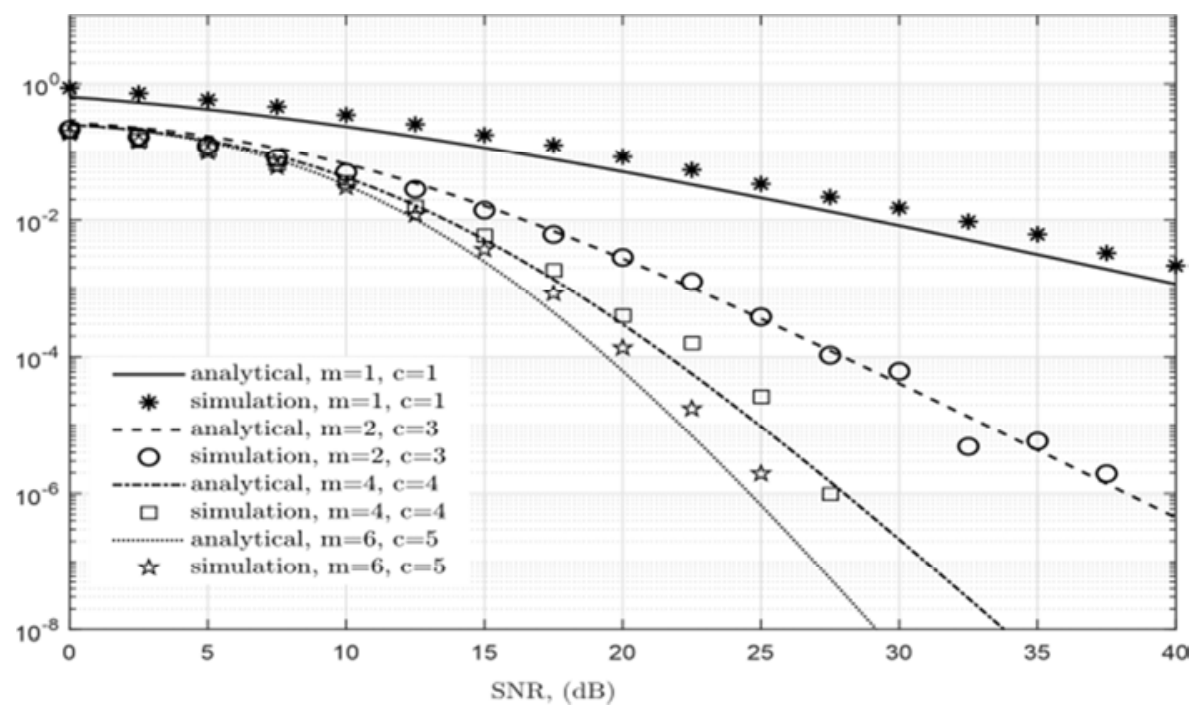

Figure 4. BEP for OFDM-IM with 16-QAM as a function of SNR over various shadowing and fading parameters. 


\section{Efficiency Metrics Enhancement Using Adaptive OFDM-IM with M-QAM Technique}

\subsection{Adaptive M-ary QAM-OFDM-IM System Model}

The block diagram of the adaptive OFDM-IM transceiver system is illustrated in Figure 1. The main concept of adaptive modulation is that the modulator and demodulator have to be configured at any time for the same constellation size $\mathrm{M}$ in the active OFDM-IM subcarriers and according to the estimated $\gamma$, which requires achieving maximum throughput as shown in Algorithm 1. Thus, depending on $\gamma$, the modulation size is chosen to satisfy a certain average bit error probability $\left(10^{-3}\right.$ or $10^{-6}$ ). The range of $\gamma$ is divided into $\mathrm{R}$ regions to determine which M-ary QAM scheme is used when the estimated $\gamma$ falls in the $\mathrm{R}^{\text {th }}$ region. Each $\mathrm{R}$ region is assigned boundaries $\left(\gamma_{\mathrm{R}}-\right.$ $\left.\gamma_{R+1}\right)$, which are specified in Table $3 . M_{R}$ represents the modulation levels. It is assumed that when $\gamma$ is below certain threshold $\gamma_{1}$, the BPSK modulation is used in the transmitter side and the channel state information is assumed to be known at the reception side.

Table 3. Switching Boundaries of $\gamma_{R}$ with Target BER $10^{-6}$ and BER $10^{-3}$.

\begin{tabular}{|c|c|c|c|}
\hline Region (R) & $\mathbf{M}_{\mathrm{R}}$ & BER $10^{-6} \gamma_{R^{-}} \gamma_{R+1}[\mathrm{~dB}]$ & BER $10^{-3} \gamma_{R^{-}} \gamma_{R+1}[d B]$ \\
\hline 1 & 4 & $13.8-17.6$ & $10.3-13.9$ \\
\hline 2 & 8 & $17.6-20.9$ & $13.9-17.2$ \\
\hline 3 & 16 & $20.9-24.0$ & $17.2-20.4$ \\
\hline 5 & 64 & $27.1-30.1$ & $23.5-26.5$ \\
\hline 6 & 128 & $30.1-\infty$ & $26.5-\infty$ \\
\hline
\end{tabular}

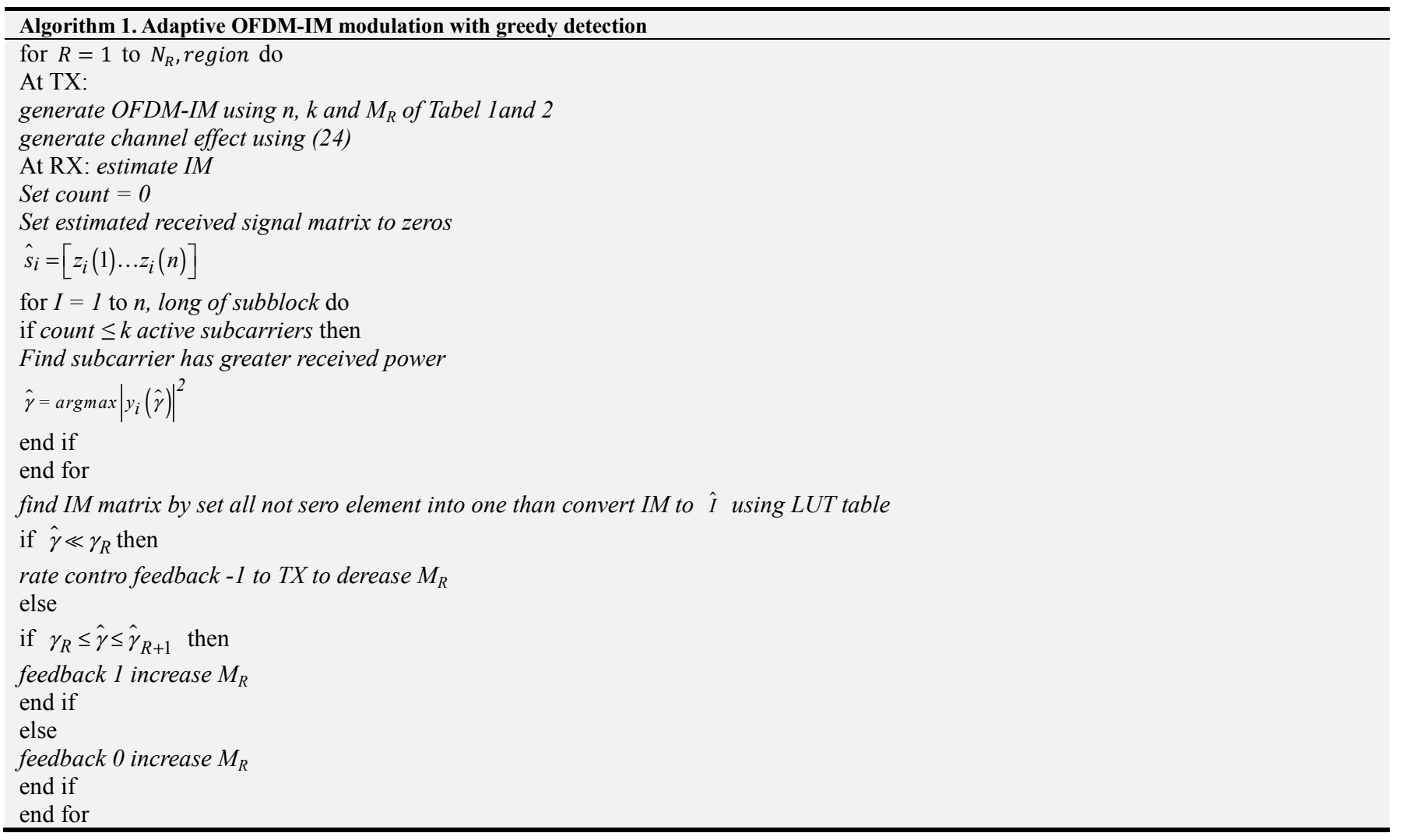

\subsection{Outage Probability}

Outage Probability, $\mathrm{P}_{\text {out }}$, also known as outage probability is the probability that the received SNR, $\gamma$ drops below the required threshold, $\gamma_{\text {th }}$ which is computed to meet the target BER of $10^{-3}$ or $10^{-6}$ as outlined in Table (3) and $P_{\text {out }}$ can be calculated using (5) as:

$$
P_{\text {out }}=\int_{0}^{\gamma_{t h}} P_{\gamma}(\gamma) d \gamma
$$

substituting (5) into (25) and using [14, (3.381.1), (8.352.6), (3.471.9)], the $P_{\text {out }}$ can be expressed as: 


$$
P_{\text {out }}=1-\frac{2}{\Gamma(c)} \sum_{i=0}^{m-1} \frac{1}{i !}\left(\beta \gamma_{t h}\right)^{\frac{c+i}{2}} K_{c-i}\left(2 \sqrt{\beta \gamma_{t h}}\right)
$$

Where $\beta=\frac{c m}{\gamma}$ and $\gamma_{\text {th }}$ is the received signal threshold.
Figure 5 illustrates the outage probability for various values of the composite shadowing and fading channel with target BERs of $10^{-3}$ and $10^{-6}$, respectively. It is observed that the outage probability $\mathrm{P}_{\text {out }}$ gets better with an increase in $\mathrm{m}$ and $\mathrm{c}$ values decreases the fading and shadowing effect on system's performance.

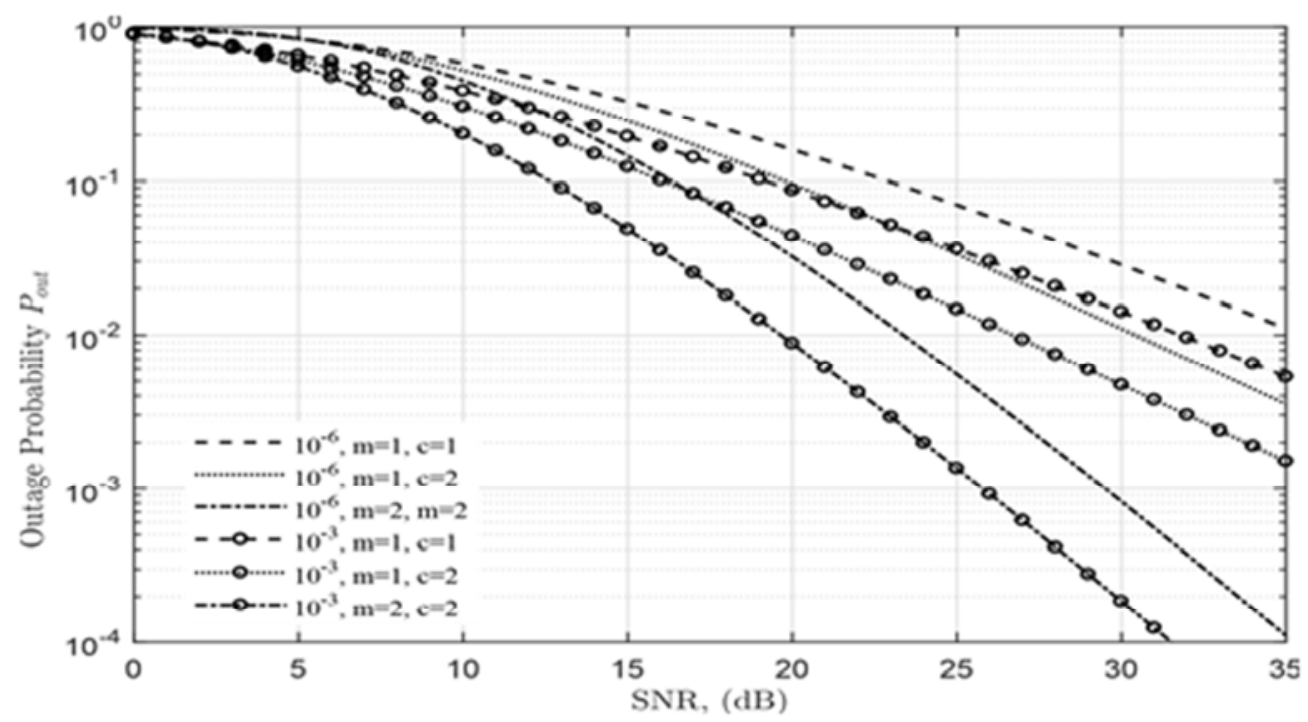

Figure 5. Outage probability target BER $10^{-6}$ and $10^{-3}$ as a function of SNR over various shadowing and fading parameters.

\subsection{Spectral and Energy Efficiency Over Fading and Shadowing Channel}

The main design parameters of a communication system are spectral efficiency (SE) and energy efficiency (EE). Both these parameters should be sustained to satisfy certain quality of service (QoS) demands. Minimizing energy consumption in wireless communication systems has become a crucial factor; however, increasing data usage leads to more energy use. By employing OFDM-IM, SE can be enhanced and at the same time EE can be reduced. In this section, the upper bounds of SE and EE for OFDM-IM are investigated:

\subsubsection{Average System Spectral Efficiency (bit/s/Hz)}

Spectral Efficiency, $\eta_{S E}$, is known as the maximum received bits per channel bandwidth (in $\mathrm{Hz}$ ) that a wireless channel can be conveyed to satisfy certain QoS requirements and it is given by [18]:

$$
\eta_{S E}=\frac{I(X ; Y)}{B T_{s}}, ? \quad b \quad s \quad H z
$$

where $B T s$ in $H z /$ baud is the bandwidth used by the symbol duration. The term $I(X, Y)$ is the achievable average data rate of the modulation scheme and it is measured by bits/channel use.

The average spectral efficiency, $\bar{\eta}_{S E}$, is the sum of the data rate $\left(\left[\log _{2} C_{n}^{k} M^{k}\right]\right)$ linked to $\mathrm{R}$ region, each weighted by the $\mathrm{n}$ probability of which that $\gamma$ falls in the $\mathrm{R}^{\text {th }}$ region and is presented by [10]:

$$
\bar{\eta}_{S E}=\sum_{R=1}^{N_{R}}\left(\left\lfloor\log _{2} C_{n}^{k} M^{k}\right\rfloor\right) P_{r}(R)
$$

Where $P_{r}(R)=\int_{\gamma_{R}}^{\gamma_{R+1}} P_{\gamma}(\gamma) d \gamma$ which can be written as:

$$
P_{r}(R)=\int_{0}^{\gamma_{R+1}} P_{\gamma}(\gamma) d \gamma-\int_{0}^{\gamma_{R}} P_{\gamma}(\gamma) d \gamma
$$

$\mathrm{P}_{\mathrm{r}}(\mathrm{R})$ can be obtained directly from (26) as:

$$
\begin{aligned}
& P_{r}(R)=\frac{2}{\Gamma(c)} \sum_{i=0}^{m-1} \frac{1}{i !}\left[\left(\beta \gamma_{R}\right)^{\frac{c+i}{2}} K_{c-i}\left(2 \sqrt{\beta \gamma_{R}}\right)-\right. \\
& \left.\left(\beta \gamma_{R+1}\right)^{\frac{c+i}{2}} K_{c-i}\left(2 \sqrt{\beta \gamma_{R+1}}\right)\right]
\end{aligned}
$$

Figure 6, Figure 7, Figure 8, and Figure 9 show the average SE (bits/s/Hz) of adaptive OFDM-IM with M-QAM modulation for four $\left(\mathrm{N}_{\mathrm{R}}=4\right)$ and five $\left(\mathrm{N}_{\mathrm{R}}=5\right)$ regions as function of SNR for various fading $(\mathrm{m})$, shadowing channel parameters (c) and active subcarriers (k). Figure 10 illustrates the average $\mathrm{SE}$ of the proposed system in three dimensions (3D) as a function of SNR for different values of $\mathrm{m}, \mathrm{c}, \mathrm{N}_{\mathrm{R}}$ and $\mathrm{k}$. It is noticed that when the adaptive scheme employs a higher constellation level the system SE is increased. For instance, system SE is enhanced by $0.5 \mathrm{bit} / \mathrm{s} / \mathrm{Hz}$ when the adaptive modulation increases the modulation level from $M=32$, i.e., $N_{R}=4$, to $M=64$, i.e., $N_{R}=5$. Moreover, the total system $\mathrm{SE}$ is improved by almost $1 \mathrm{bit} / \mathrm{s} / \mathrm{Hz}$ as the active subcarriers (k) are increased. Also, adaptive modulation offers better adaptation with any impaired channel conditions. 


\subsubsection{Average System Energy Efficiency (bits/TNEU)}

Energy efficiency, $\eta_{E E}$, is defined as the maximum data rate $(R)$ in $(b i t / s)$ that can be received by wireless communication systems per unit of transmitted power, $p_{t}$, and that is [19]:

$$
\eta_{E E}=\frac{R}{P_{t}}, \quad(\text { bit } / s / \text { Watt })
$$

Another definition, including the effect of wireless channel energy efficiency, has been presented as the number of bits per thermal noise signal energy unit (TNEU). Therefore, energy efficiency is associated with SNR, $\gamma=p_{t} / B N_{0}$, and it can be expressed as [20]:

$$
\eta_{E E}=\frac{\eta_{s}}{\gamma}, \quad(b / T E N U)
$$

When the SE is achieved, the EE of adaptive transmission is directly obtained from (23) as:

$$
\bar{\eta}_{E E}=\frac{\bar{\eta}_{S E}=\sum_{R=1}^{N_{R}}\left(\left\lfloor\log _{2} C_{n}^{k} M^{k}\right\rfloor\right) P_{r}(R)}{\gamma}
$$

The EE is shown in Figure 11 and Figure 12. Figure 13 illustrates the EE as a function of SNR for two regions $(\mathrm{NR}=$ 4 and 5), with $\mathrm{c}=4, \mathrm{~m}=3$ and various values $\mathrm{k}$. It is obvious that the $\mathrm{EE}$ is gets better as the channel effect decreases. Also, the EE is improved as the number of active subcarriers (k) increases. For example, in the case of $\mathrm{m}=1$ and $\mathrm{c}=1$, the EE is enhanced by $13 \%$ when the active subcarrier is reduced from $\mathrm{k}=3$ to $\mathrm{k}=2$. Also, it is clear from Figure 13 that the lower NR provides better EE by $15 \%$.

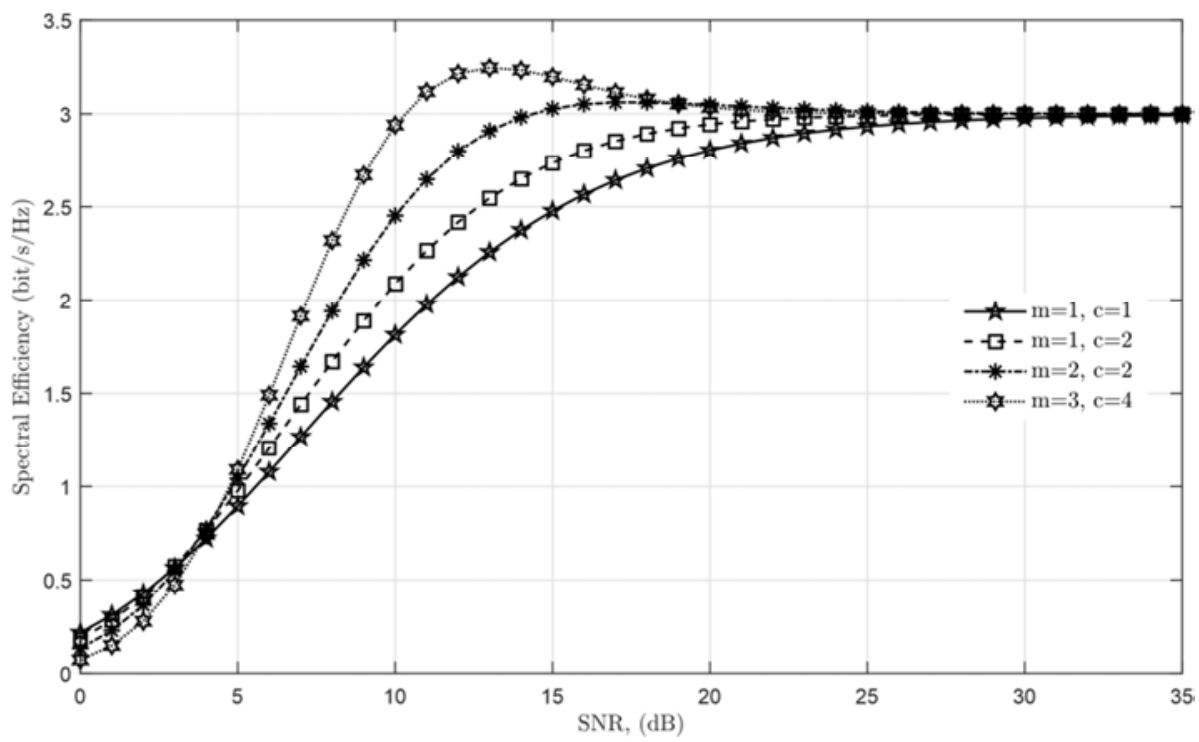

Figure 6. Spectral efficiency of adaptive M-QAM for OFDM-IM over shadowing and fading channels as a function of SNR for NR=4, $k=2, n=4$ and different values of $m$ and $c$.

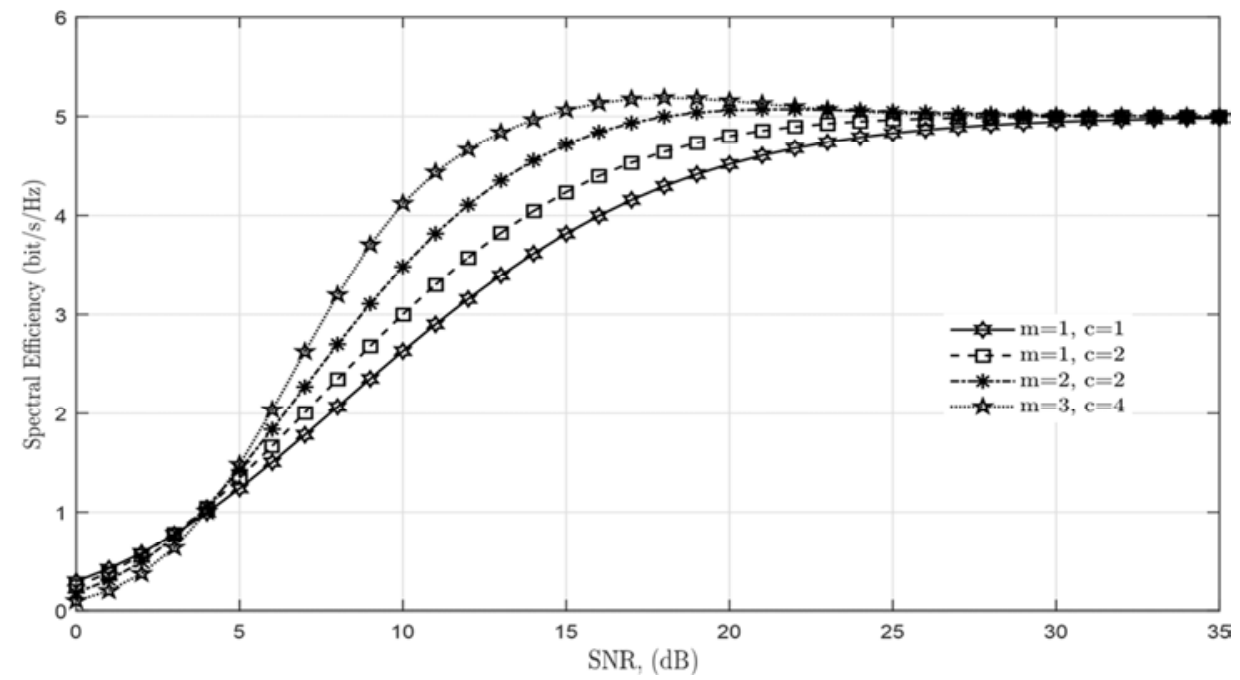

Figure 7. Spectral efficiency of adaptive M-QAM for OFDM-IM over shadowing and fading channels as a function of SNR for $N_{R}=4, k=3, n=4$ and different values of $m$ and $c$. 


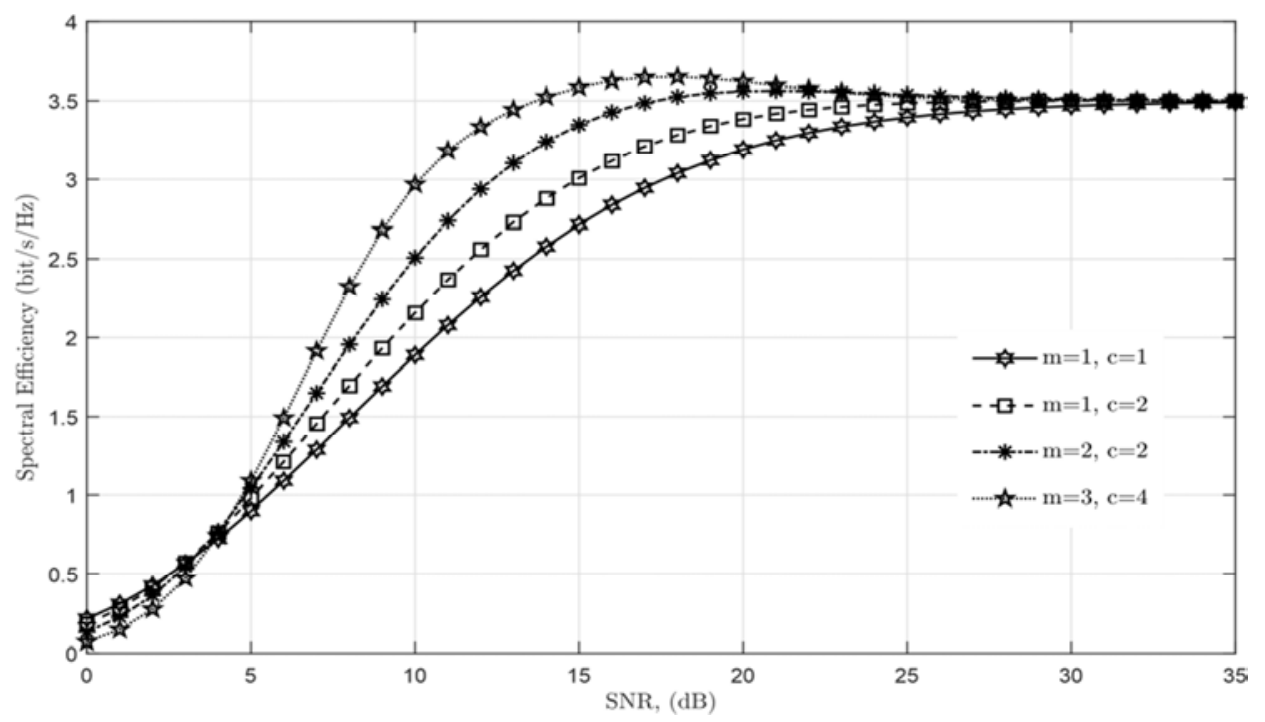

Figure 8. Spectral efficiency of adaptive M-QAM for OFDM-IM over shadowing and fading channels as a function of SNR for $N_{R}=5, k=2, n=4$ and different values of $m$ and $c$.

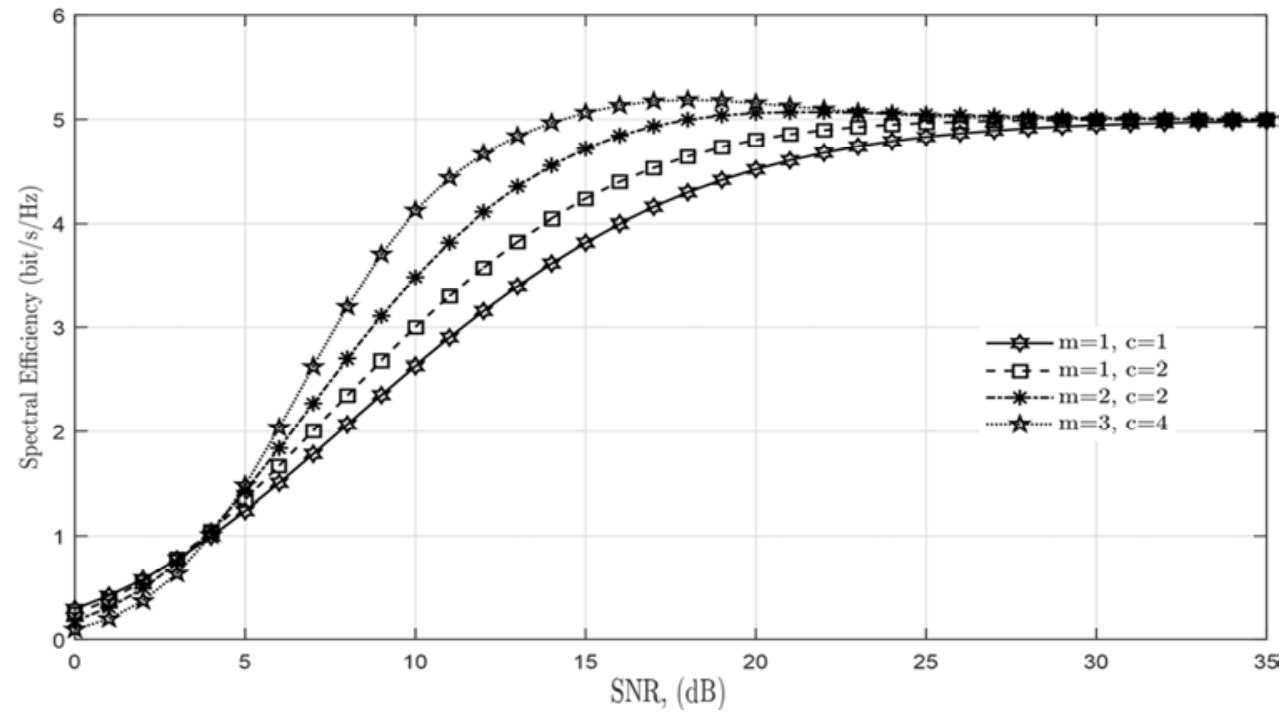

Figure 9. Spectral efficiency of adaptive M-QAM for OFDM-IM over shadowing and fading channels as a function of SNR for $N_{R}=5, k=3, n=4$ and different values of $m$ and $c$.

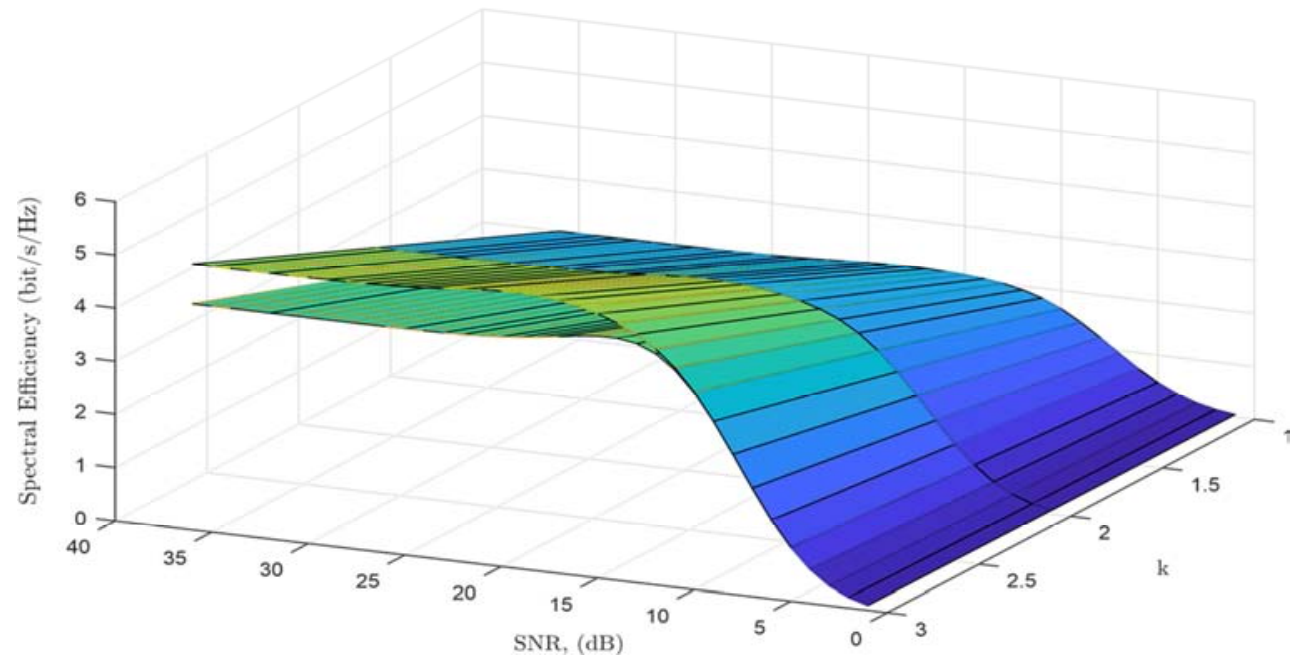

Figure 10. Spectral efficiency of adaptive M-QAM for OFDM-IM over shadowing and fading channels as a function of SNR for $m=3, c=4, n=4$ and different values of $N_{R}$ and $k$. 


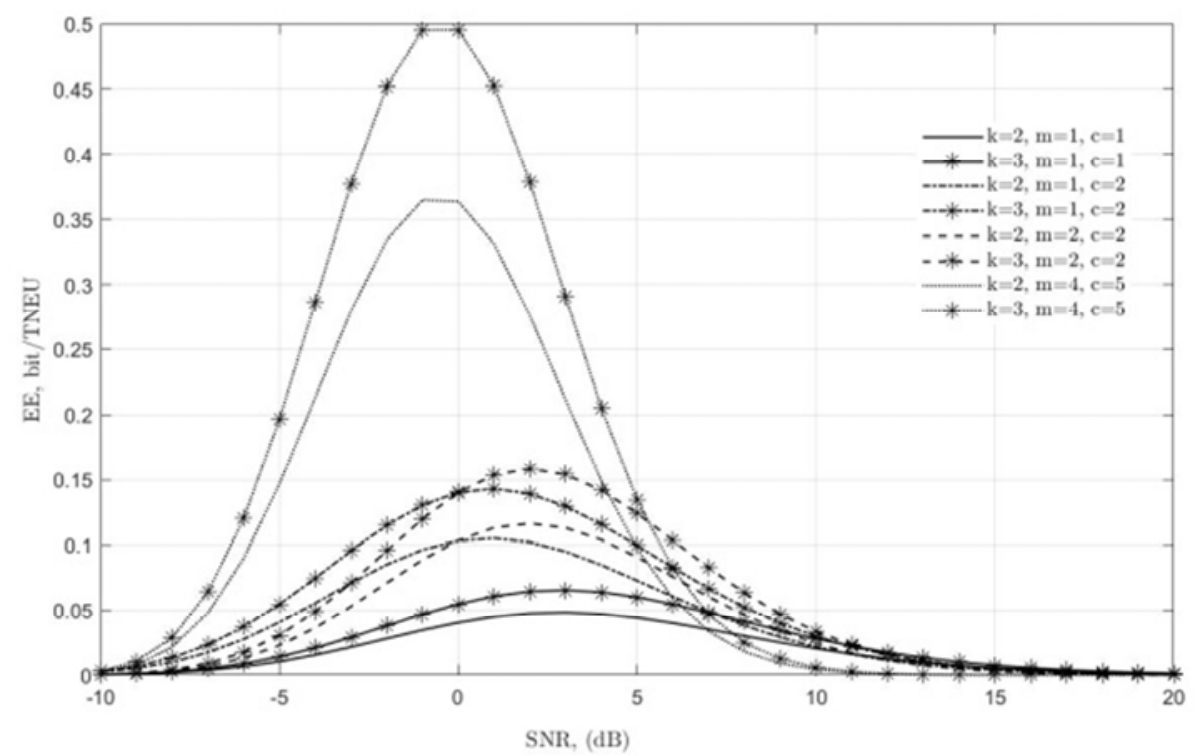

Figure 11. Energy efficiency of adaptive M-QAM for OFDM-IM over shadowing and fading channels as a function of $S N R$ for $N_{R}=4, n=4$ and different values of $c, m$ and $k$.

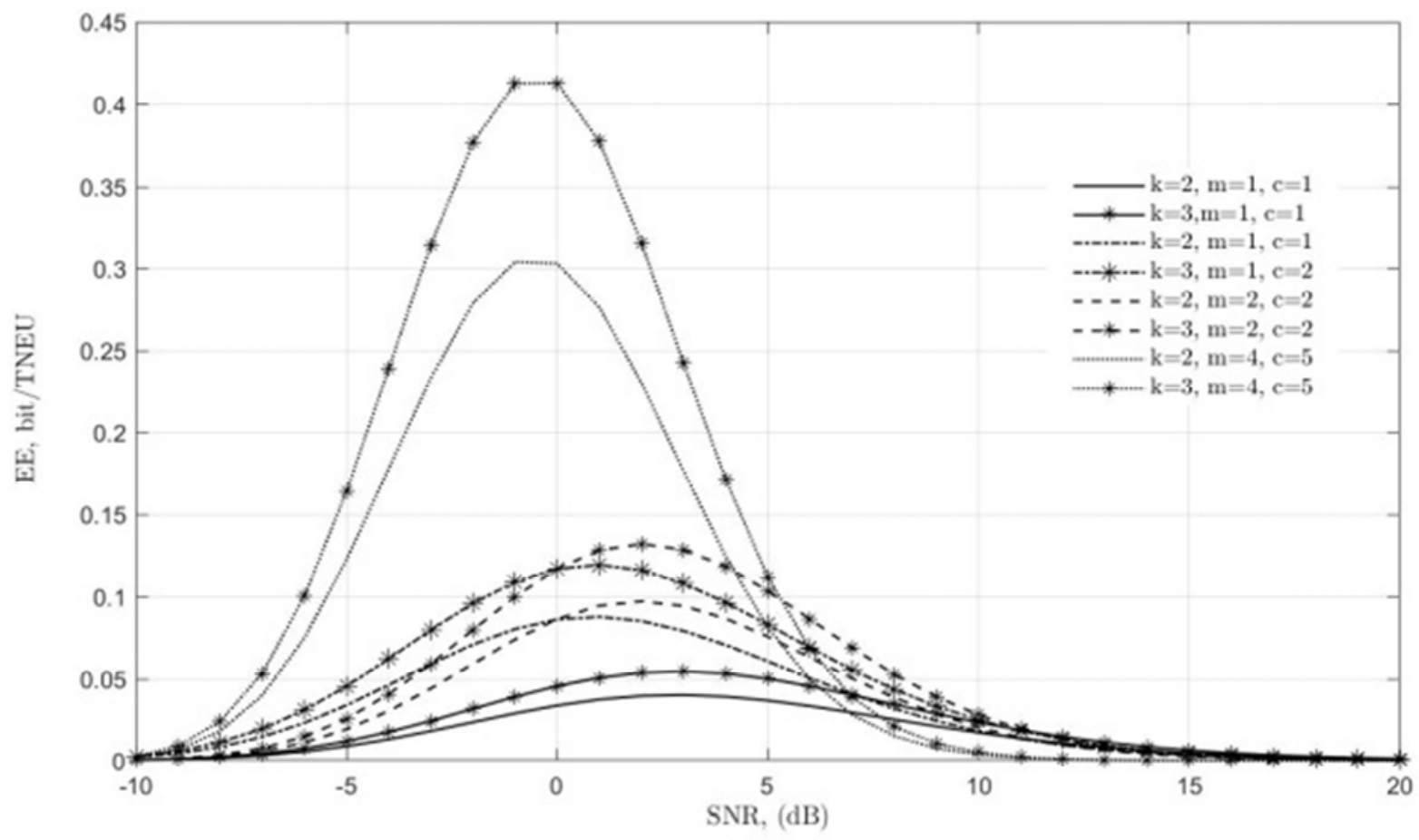

Figure 12. Energy efficiency of adaptive M-QAM for OFDM-IM over shadowing and fading channels as a function of SNR for $N_{R}=5, n=4$ and different values of $c, m$ and $k$.

\subsection{Average Bit Error Probability (ABEP)}

The presented adaptive Modulation with OFDM-IM schemes is designed based on separated SNR regions and it aims to maximize EE and SE by changing the active subcarriers $(\mathrm{k})$ in each sub-block (n). However, it must maintain a certain QoS, which is measured in terms of ABEP. Therefore, ABEP can be determined by averaging the BEP for all $\gamma$ regions, and this can be computed exactly as the ratio of the average number of bits in error over the average total number of transmitted bits as [10]:

$$
A B E P=\frac{\sum_{R=1}^{N_{R}}\left(\left\lfloor\log _{2}\left(M_{R}^{k} C_{n}^{k}\right)\right\rfloor\right) \overline{B E P}_{R}}{\overline{\eta_{S E}}=\sum_{R=1}^{N_{R}}\left(\left\lfloor\log _{2}\left(M_{R}^{k} C_{n}^{k}\right)\right\rfloor\right) P_{r}(R)},
$$

where $\overline{B E P}_{R}$ is the average bit error probability over composite fading/shadowing channel for $R^{\text {th }}$ region and can be attained using: 


$$
\overline{B E P}_{R}=\int_{\gamma_{R}}^{\gamma_{R+1}} B E P \cdot P_{\gamma}(\gamma) d \gamma
$$

where $P_{\gamma}(\gamma)$ is given by (6) and $B E P$ is expressed by (16). Thus, $\overline{B E P}_{R}$ can be written as:

$$
\begin{aligned}
& \overline{B E P}_{R}=\frac{k}{n} \sum_{\delta=1}^{n}[\underbrace{\int_{\gamma_{R}}^{\gamma_{R+1}} P E P \cdot P_{\gamma}(\gamma) d \gamma}_{\overline{B E P}_{R}^{1}} \\
& -\underbrace{\int_{\gamma_{R}}^{\gamma_{R+1}} P_{e_{M}} \cdot P_{\gamma}(\gamma) d \gamma}_{\overline{B E P}_{R}^{2}} \\
& +\underbrace{\int_{\gamma_{R}}^{\gamma_{R+1}} P E P \cdot P_{e_{M}} \cdot P_{\gamma}(\gamma) d \gamma}_{\overline{B E P}_{R}^{3}}]
\end{aligned}
$$

By comparing (7) and (8) with (33) and with the aid of [14, (3.381.1), (8.352.6), (3.471.9)], the overall upper bound and approximate of $\overline{B E P}_{R}$ can be expressed as:

$$
\begin{aligned}
& \overline{B E P}_{R}=\frac{k}{n} \sum_{\delta=1}^{n}\left[\overline{B E P}_{R}^{1}+\overline{B E P}_{R}^{2}+\overline{B E P}_{R}^{3}\right] \\
& =k\left[\overline{B E P}_{R}^{1}+\overline{B E P}_{R}^{2}+\overline{B E P}_{R}^{3}\right]
\end{aligned}
$$

The three different bit error probabilities $\left(\mathrm{BEP}_{\mathrm{R}}{ }^{1}, \mathrm{BEP}_{\mathrm{R}}{ }^{2}\right.$, $\mathrm{BEP}_{\mathrm{R}}{ }^{3}$ ) of exact and approximate upper bound in (34) for all $R^{\text {th }}$ regions are evaluated as:

$$
\begin{aligned}
& \overline{B E P}_{R, \text { approx }}^{1}=\frac{(n-k) \beta^{l_{1}}}{\Gamma(c)}\left(\frac{1}{2}\right)^{-m}\left[\gamma_{R}^{l} e^{-\frac{\gamma_{R}}{2}} \sum_{i=0}^{m-1} \frac{1}{i !}\right. \\
& \left(\frac{\gamma_{R}}{2}\right)^{i} K_{c-m}\left(2 \sqrt{\beta \gamma_{R}}\right)-\gamma_{R+1} e^{-\frac{\gamma_{R+1}}{2}} \sum_{i=0}^{m-1} \frac{1}{i !}\left(\frac{\gamma_{R+1}}{2}\right)^{i} \\
& \left.K_{c-m}\left(2 \sqrt{\beta \gamma_{R+1}}\right)\right] \\
& \overline{B E R}_{R, a p p r o x}^{2}=\frac{0.4 \beta^{l_{1}}(\alpha)^{-m}}{\Gamma(c)}\left[\gamma_{R}^{l} e^{-\frac{\gamma_{R}}{\alpha}} \sum_{i=0}^{m-1} \frac{1}{i !}\left(\frac{\gamma_{R}}{\alpha}\right)^{i}\right. \\
& K_{c-m}\left(2 \sqrt{\beta \gamma_{R}}\right)-\gamma_{R+1}^{l} e^{-\frac{\gamma_{R+1}}{\alpha}} \sum_{i=0}^{m-1} \frac{1}{i !}\left(\frac{\gamma_{R+1}}{\alpha}\right)^{i} \\
& \left.K_{c-m}\left(2 \sqrt{\beta \gamma_{R+1}}\right)\right]
\end{aligned}
$$

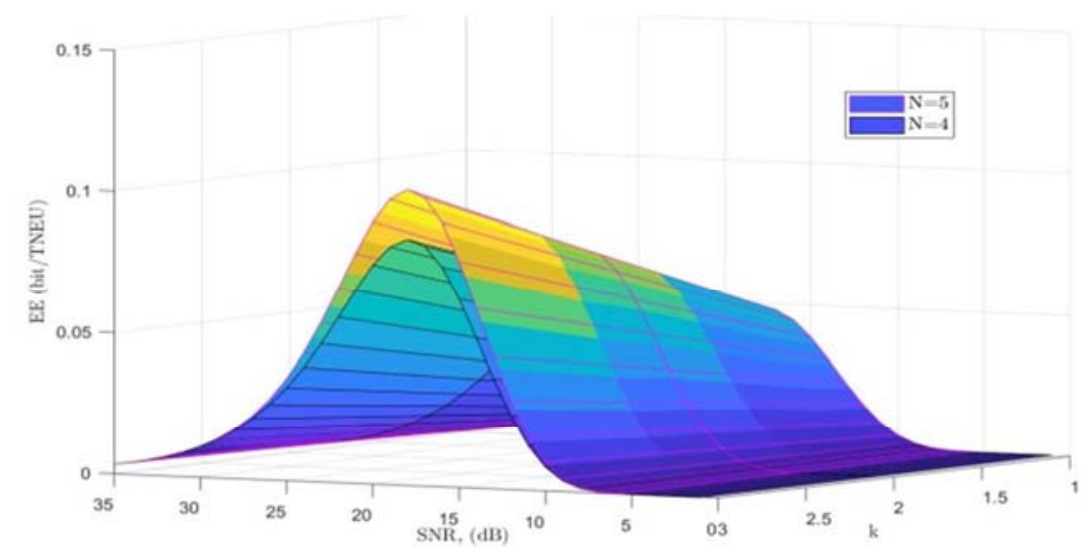

Figure 13. Energy efficiency of adaptive M-QAM for OFDM-IM over shadowing and fading channels as a function of $S N R$ for $N_{R}=4, N_{R}=5$, with $c=4, m=3$, $n=4$ and various values $k$.

$$
\begin{aligned}
& \overline{B E R}_{R, \text { approx }}^{3}=\frac{0.4 \beta^{l_{1}}}{\Gamma(c)}\left(\frac{D}{2}\right)^{-m}\left[\gamma_{R}^{l} e^{-\frac{D \gamma_{R}}{2}} \sum_{i=0}^{m-1} \frac{1}{i !}\left(\frac{D \gamma_{R}}{2}\right)^{i}\right. \\
& K_{c-m}\left(2 \sqrt{\beta \gamma_{R}}\right)-\gamma_{R+1}^{l} e^{-\frac{D \gamma_{R+1}}{2}} \sum_{i=0}^{m-1} \frac{1}{i !}\left(\frac{D \gamma_{R+1}}{2}\right)^{i} \\
& \left.K_{c-m}\left(2 \sqrt{\beta \gamma_{R+1}}\right)\right] \\
& \overline{B E P}_{R \text {,exact }}=\frac{2}{\Gamma(c)} \sum_{i=0}^{m-1} \frac{1}{i !}\left[\left(\beta \gamma_{n}\right)^{\frac{c+i}{2}} K_{c-i}\left(2 \sqrt{\beta \gamma_{n}}\right)\right. \\
& \left.-\left(\beta \gamma_{n+1}\right)^{\frac{c+i}{2}} K_{c-i}\left(2 \sqrt{\beta \gamma_{n+1}}\right)\right]-\frac{2}{\Gamma(c)} \sum_{q=0}^{n-k}\left(\begin{array}{c}
n-k \\
q
\end{array}\right) \\
& \frac{-1^{q}}{q+1} \beta^{l_{1}} L^{-m} \sum_{i=0}^{m-1}\left[\gamma_{n}\left(L \gamma_{n}\right)^{i} e^{-L \gamma_{n}} K_{c-m}\left(2 \sqrt{\beta \gamma_{n}}\right)\right. \\
& \left.-\gamma_{n+1}\left(L \gamma_{n+1}\right)^{i} e^{-L \gamma_{n+1}} K_{c-m}\left(2 \sqrt{\beta \gamma_{n+1}}\right)\right] \\
& \overline{B E P}_{R, \text { exact }}^{2}=\frac{0.4(1 / k-1)}{\Gamma(c)} \beta^{1} \alpha^{n}\left[\gamma_{n} e^{\frac{\gamma_{n}}{\alpha}} \sum_{i=0}^{m-1} \frac{1}{i !}\left(\frac{\gamma_{n}}{\alpha}\right)^{i}\right. \\
& K_{c-m}\left(2 \sqrt{\beta \gamma_{n}}\right)-\gamma_{n+1} e^{\frac{\gamma_{n+1}}{\alpha}} \sum_{i=0}^{m-1} \frac{1}{i !}\left(\frac{\gamma_{n+1}}{\alpha}\right)^{i=} \\
& \left.K_{c-m}\left(2 \sqrt{\beta \gamma_{n+1}}\right)\right] \\
& \overline{B E P}_{R, \text { exact }}^{3}=-\frac{0.4}{\Gamma(c)} \beta^{h_{1}} \sum_{q=0}^{n-k}\left(\begin{array}{c}
n-k \\
q
\end{array}\right) \frac{-1}{q+1}\left(L+\frac{1}{\alpha}\right)^{-m} \\
& {\left[\gamma_{n} e^{\left(L+\frac{1}{\alpha}\right) \gamma_{n}} \sum_{i=0}^{m-1} \frac{1}{i !}\left(L \gamma_{n}+\frac{\gamma_{n}}{\alpha}\right) K_{c-m}\left(2 \sqrt{\beta \gamma_{n}}\right)-\gamma_{n+1}\right.} \\
& \left.e^{\left(L+\frac{1}{\alpha}\right) \gamma_{n+1}} \sum_{i=0}^{m-1} \frac{1}{i !}\left(L \gamma_{n+1}+\frac{\gamma_{n+1}}{\alpha}\right) K_{c-m}\left(2 \sqrt{\beta \gamma_{n+1}}\right)\right]
\end{aligned}
$$

$A B E P$ of the proposed OFDM-IM with M-QAM modulation is obtained form (32) for the $\mathrm{N}_{\mathrm{R}}$ regions. Figure 14 and Figure 15 illustrate ABEP of $10^{-3}$ and $10^{-6}$ as a function of SNR for $N_{R}=4, k=2, n=4$ and various values of $m$ and $c$, respectively. Figure 16 depicts the effects of different values of $k$ and channel parameters. It is evident that the adaptive OFDM-IM with M-QAM modulation preserves a certain QoS for all the values of $m$ and $c$ in the targeted $\gamma$ range. Also, Figure 16 shows the effect of increasing $k$ in $A B E P$ for various values of channel parameters. Here, for high values of $\gamma$, the performance of the system is controlled by M-QAM modulation levels. 


\section{Conclusion}

The performance of OFDM-IM system has been investigated in a multi-path fading channel superimposed on a shadowing environment, by using composite Nakagami-m-Gamma (NG) model. The closed-form expressions for $P E P$ and $A B E P$ have been derived over the proposed channel and validated using Monte Carlo simulation. Also, the closed-form boundaries of SE and EE for OFDM-IM with the M-QAM modulation scheme have been examined in regards to active subcarriers $(k), c$ and $m$ channel parameters, and SNR. We observe that SE and EE are highly dependent on the channel parameters. Thus, the adaptive modulation scheme was proposed to improve the efficiency of OFDM-IM system with M-QAM modulation scheme. The performance of the adaptive modulation with OFDM-IM system has been investigated in terms of outage probability, average SE, average EE, and average bit error probability. The results show that by varying the number of active subcarriers (k), the proposed adaptive OFDM-IM system not only enhances the SE and EE, but also.

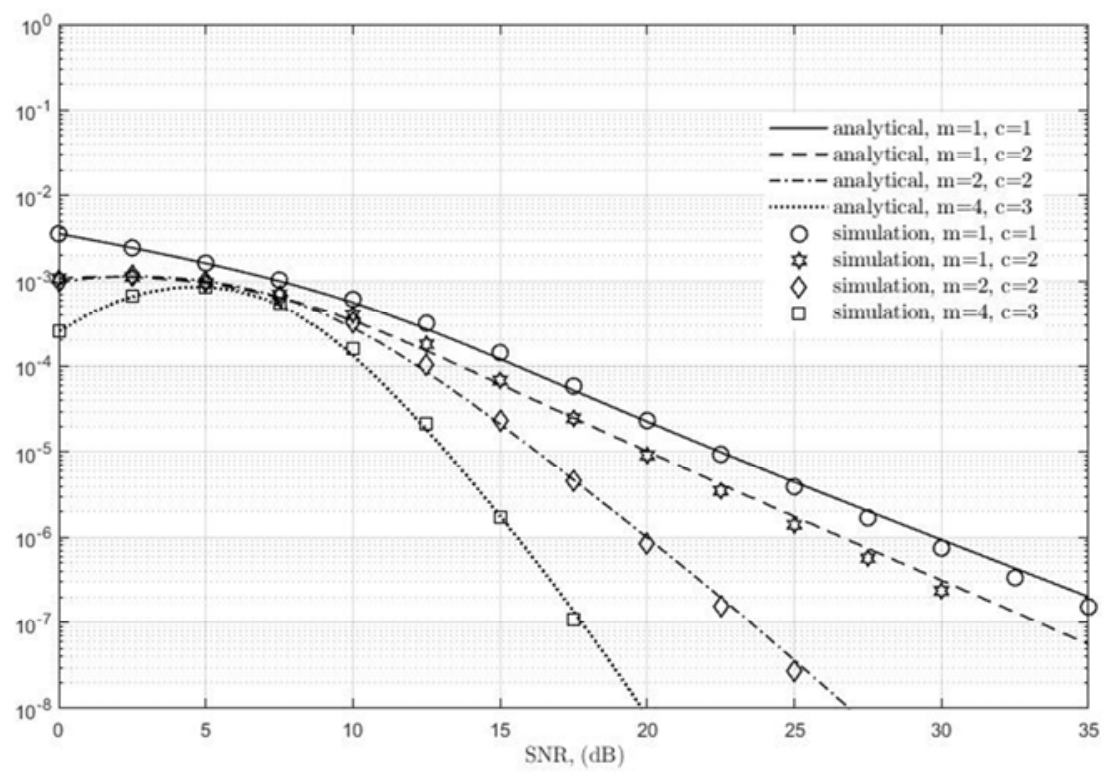

Figure 14. AEBP of adaptive M-QAM for OFDM-IM over shadowing and fading channels as a function of $S N R$ for $N_{R}=4, n=4$ and different values of $k, m$ and c targeting $10^{-3}$.

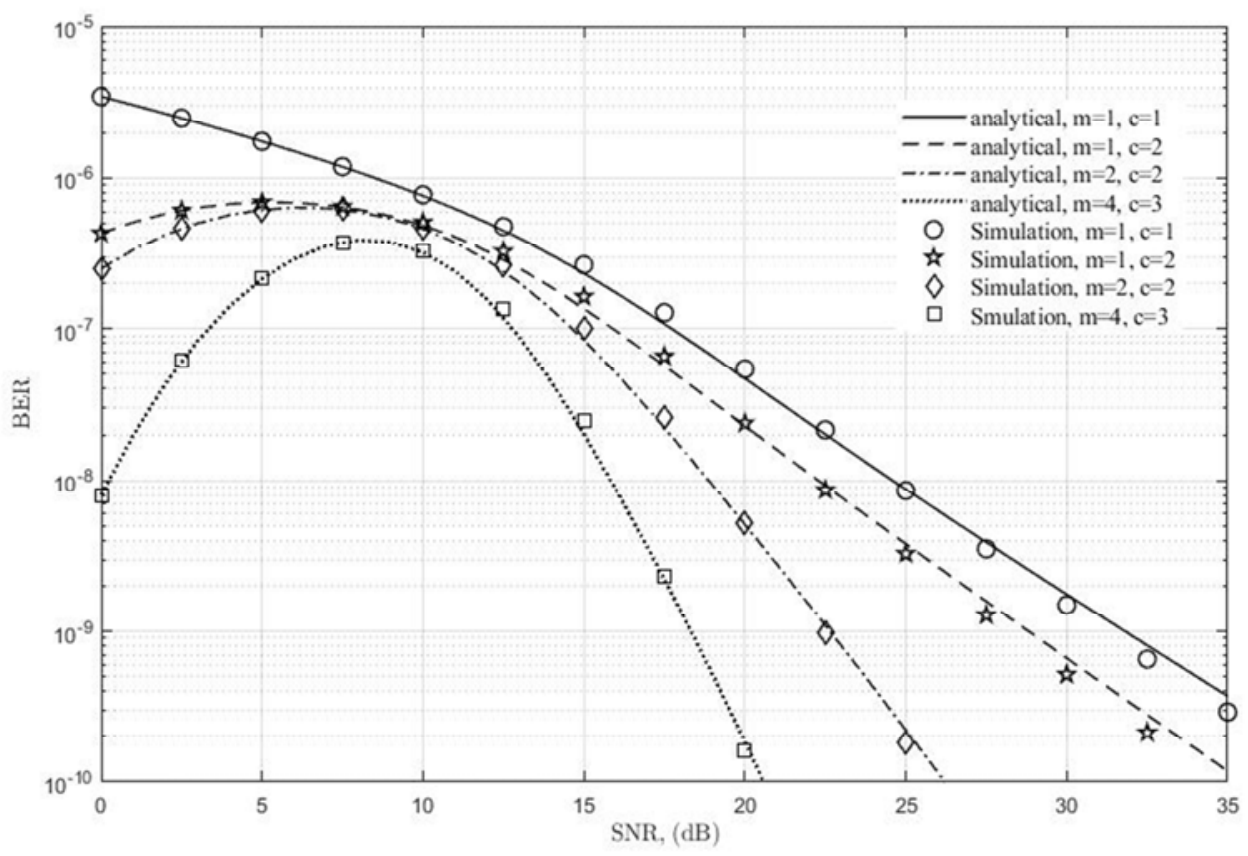

Figure 15. AEBP of adaptive M-QAM for OFDM-IM over shadowing and fading channels as a function of $S N R$ for $N_{R}=4, n=4$ and different values of $k, m$ and c targeting $10^{-6}$. 


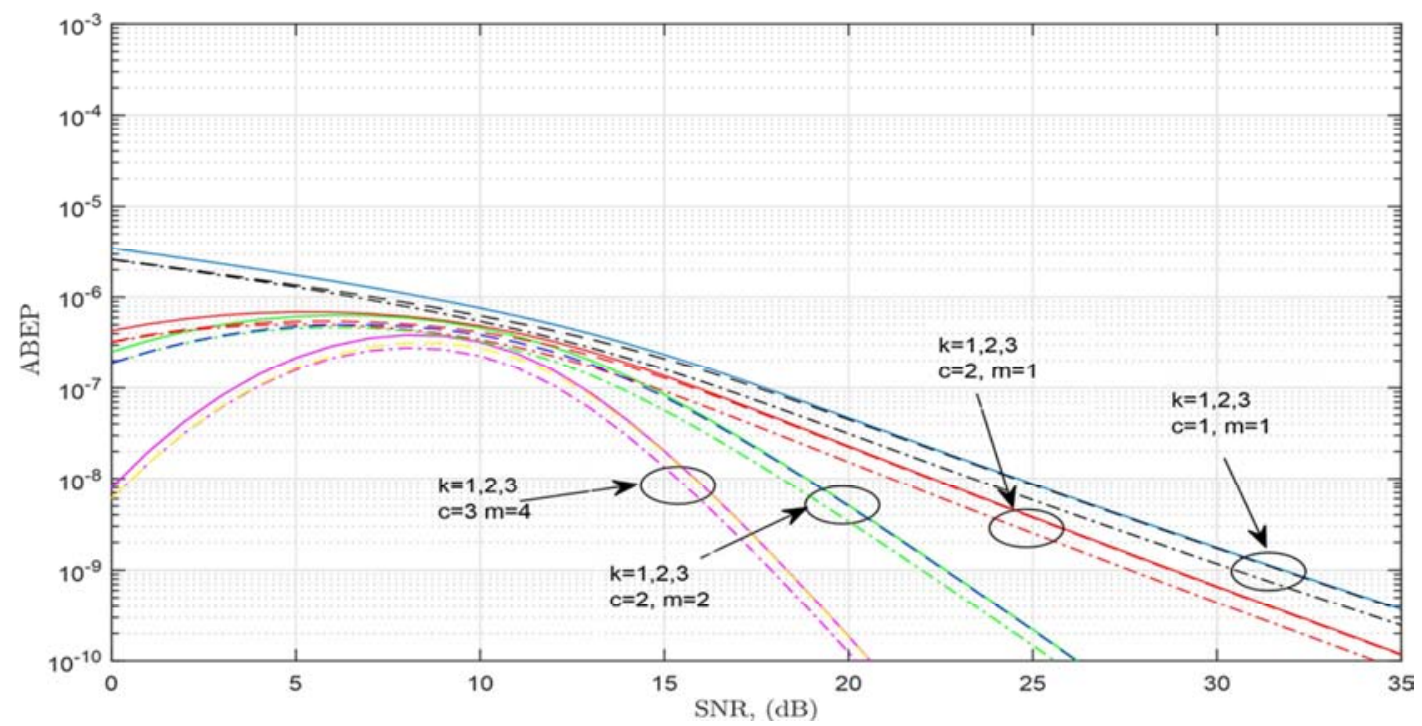

Figure 16. AEBP of adaptive M-QAM for OFDM-IM over shadowing and fading channels as a function of $S N R$ for $N_{R}=4, k=2, n=4$ and different values of $m$ and c targeting $10^{-6}$.

\section{Acknowledgements}

The authors would like to express gratitude to the Libyan Ministry of Higher Education for their support in carrying of this research work.

\section{References}

[1] R. Abu-Alhiga and H. Haas, "Subcarrier-index modulation ofdm," In Personal, Indoor and Mobile Radio Communications, 2009 IEEE 20th International Symposium on. IEEE, 2009, pp.

[2] D. Tsonev, S. Sinanovic, and H. Haas, "Enhanced subcarrier index modulation (SIM) OFDM," in GLOBECOM Workshops (GC Wkshps), 2011 IEEE. IEEE, 2011, pp. 728-732.

[3] Başar, Ertuğrul, et al. "Orthogonal frequency division multiplexing with index modulation." IEEE Transactions on Signal Processing 61.22 (2013): 5536-5549.

[4] R. Fan, Y. J. Yu, and Y. L. Guan, "Orthogonal frequency division multiplexing with generalized index modulation," in Global Com $\neg$ munications Conference (GLOBECOM), 2014 IEEE. IEEE, 2014, pp. 3880-3885.

[5] Y. Ko, "A tight upper bound on bit error rate of joint ofdm and multi-carrier index keying," IEEE Communications Letters, vol. 18, no. 10, pp. 1763-1766, Oct 2014. M. Young, The Technical Writer's Handbook. Mill Valley, CA: University Science, 198.

[6] J. Crawford and Y. Ko, "Low complexity greedy detection method with generalized multicarrier index keying ofdm," in 2015 IEEE 26th Annual International Symposium on Personal, Indoor, and Mobile Radio Communications (PIMRC), Aug 2015, pp. 688-693.

[7] E. Chatziantoniou, J. Crawford, and Y. Ko, "Performance analysis of a low-complexity detector for mcik-ofdm over twdp fading," IEEE Communications Letters, vol. 20, no. 6, p. Eleftherios, June 2016.
[8] I. Aboharba, H. Boud, Q. M. Rahman, and R. K. Rao, "Performance analysis of a low-complexity detection for OFDM Index Modulation over Nakagami-m fading," in 2017 IEEE 30th Canadian Conference on Electrical and Computer Engineering (CCECE), April 2017, pp. 1-4.

[9] I. Aboharba, H. Boud, Q. M. Rahman, and R. K. Rao, "On the performance of ofdm index modulation over nakagami fading channels," in 2017 IEEE 30th Canadian Conference on Electrical and Computer Engineering (CCECE), April 2017, pp. $1-5$.

[10] M.-S. Alouini and A. J. Goldsmith, "Adaptive modulation over nakagami fading channels," Wireless Personal Communications, vol. 13, no. 1-2, pp. 119-143, 2000.

[11] Simon, Marvin K., and Mohamed-Slim Alouini. "Digital communication over fading channels," Vol. 95. John Wiley \& Sons, 2005 .

[12] Shankar, P. M. "A Nakagami-N-gamma model for shadowed fading channels." Wireless Personal Communications 64.4 (2012): 665-680. 353-355, 2006.

[13] Shankar, P. Mohana. "Fading and shadowing in wireless systems," Springer, 2017.

[14] Gradshteyn, Izrail Solomonovich, and Iosif Moiseevich Ryzhik. "Table of integrals, series, and products," Academic press, 2014.

[15] Kostić, I. M. "Analytical approach to performance analysis for channel subject to shadowing and fading." IEE Proceedings-Communications 152.6 (2005): 821-827.

[16] Hamed, M. Alsharef, and R. K. Rao, "Analysis of energy and spectral efficiency in urban shadowing environment." IAENG International Journal of Computer Science, vol. 43, no. 2, 2016.

[17] V. Rodoplu and T. H. Meng, "Bits-per-joule capacity of energy-limited wireless networks," IEEE Transactions on Wireless Communications, vol. 6, no. 3, 2007. 
[18] L. Zhao, J. Cai, and H. Zhang, "Radio-efficient adaptive modulation and coding: Green communication perspective," in Vehicular Technology Conference (VTC Spring), 2011 IEEE 73rd. IEEE, 2011, pp. 1-5.

[19] A. M. Hamed, A. Noorwali, and R. K. Rao, "Energy efficient adaptive transmission in generalized-k fading channel," in
2016 IEEE Canadian Conference on Electrical and Computer Engineering (CCECE), May 2016, pp. 1-5.

[20] D. R. Iskander and A. M. Zoubir, "Estimation of the parameters of the k-distribution using higher order and fractional moments [radar clutter]," IEEE Transactions on Aerospace and Electronic Systems, vol. 35, no. 4, pp. 1453-1457, 1999. 